\title{
Variations of HRV Analysis in Different Approaches
}

\author{
FC Chang ${ }^{1}, \mathrm{CK} \mathrm{Chang}^{2}, \mathrm{CC} \mathrm{Chiu}^{1,2}, \mathrm{SF} \mathrm{Hsu}^{3}, \mathrm{YD} \mathrm{Lin}^{2}$ \\ ${ }^{1}$ Graduate Institute of Electrical and Communications Engineering, Feng Chia University, Taichung, \\ Taiwan \\ ${ }^{2}$ Department of Automatic Control Engineering, Feng Chia University, Taichung, Taiwan \\ ${ }^{3}$ Acupuncture Research Center, China Medical University, Taichung, Taiwan
}

\begin{abstract}
The analysis of normal-to-normal (NN) intervals acquired from a continuous electrocardiogram (ECG) record is a standard method to evaluate the variations in heart rate. For the advantage of responding to the pumping action of the heart, photoplethysmography $(P P G)$ has also been used extensively in the analysis of heart rate variability (HRV). However, there is little literature available on the variation between the $H R V$ analysis derived from ECG and PPG. In the research described here, experiments of recording short-term $(\leq 5$ minutes) ECG and PPG signals simultaneously from healthy subjects (male, $N=10$ ) under control were carried out to investigate the possibility of such variation. Automatic computer analysis is provided for the analysis of correlation coefficient and the $L F / H F$ ratio by autoregressive (AR) spectral analysis for evenly resampled sequences. The identical results are highly expected. However, the correlation coefficient between $R R I$ ( $R$-R interval from ECG) and PPI (peak-to-peak interval from $P P G$ ) is $0.86 \pm 0.15$, which should be unity for perfectly matched patterns. In additions, the relative $L F / H F$ ratios are $2.49 \pm 1.13$ (for ECG) and $2.73 \pm 0.82$ (for $P P G$ ) respectively. Though there is no statistical difference, the worst likelihood ratio $(L R)$ reaches the deviation of $19.04 \%$. From the experimental results, it can be appreciated that there is indeed variation for $H R V$ analysis in two different approaches even for healthy subjects under well-controlled conditions. For abnormal subjects in clinical applications, such variation may be expected to become more apparent. Though the variation is minor, it is suggested to obey the standard measure of HRV proposed by Task Force for consistent conclusions.
\end{abstract}

\section{Introduction}

Heart rate has a close relationship to sympathetic and parasympathetic nervous systems. HRV is an important index to assess and monitor cardiovascular diseases or symptoms such as arrhythmia, coronary artery diseases, myocardial infarction, diseases, hypertension, etc [1]. Heart rate signal is usually derived from electrocardiogram (ECG) [1].

Another promising approach for acquiring heart rate is photoplethysmography (PPG). The principle of PPG is that the detected light reflected from or transmitted through the artery emanating from an infrared (IR) light source will change with the pulsatile blood flow in the artery [2]. Therefore, the amplitude of PPG signal changes with time due to the cyclic change of blood volume in arteries during circulation. According to previous researches, PPG can help to evaluate sympathetic tone, cardiovascular function and respiratory volume [3-5]. PPG has the potential to monitor HR and HRV if the PPG signal is stable enough [6-9].

However, few researches have been done on whether any difference exists between ECG and PPG regarding HR and HRV. The main purpose of this study is to investigate the possible variation between the HRV analysis derived from ECG and PPG. The negative result may extend the applications of PPG in clinical diagnosis. This paper is organized as follows. The materials and methods utilized in this research will be introduced in section 2. Section 3 includes the results and related discussion. Finally, the conclusion is given in section 4.

\section{Materials and methods}

\subsection{Materials}

Ten healthy subjects (all males aged from 25 to 35, and no prior history of cardiovascular disease) took part in this study. The subjects are asked to be free of coffee and alcoholic drinks at least three hours before each experiment. The laboratory environment is kept at room temperature, and the subjects are posed in a sitting position during the experiments. Lead II ECG and PPG signals from the index fingers (left and right) are acquired simultaneously for each subject under the controlled 
conditions. The sampling frequency for both signals is $250 \mathrm{~Hz}$. Only those high-quality signals with lasting over one minute are adopted for further analysis.

The measurement device is MP30 (BIOPAC System Inc.), and the utilized sensor modules are SS2L for ECG and SS4L (with 860nm wavelength) for PPG measurement, respectively. The configuration for data acquisition is according to the assignments embedded in the package Biopac Student Lab Lessons \& PRO for PC Version3.6.7 (BIOPAC System Inc.). The acquired signals are then analyzed in LabVIEW and MATLAB environment.

\subsection{Data analysis}

To reduce the high-frequency interference and avoid the phase distortion, all of the raw ECG and PPG signals are conditioned by a DC-35Hz FIR filter before further analysis. The filtered signals are then used to acquire $\mathrm{HR}_{\mathrm{ECG}}$ (heart rate derived from ECG signal) and $\mathrm{HR}_{\mathrm{PPG}}$ (heart rate derived from PPG signal). So and Chan's algorithm is adopted for QRS detection [10], the nonevenly R-R interval sequences are then linearly interpolated according to the research by Berger et al (the sampling interval $\Delta t$ equals $0.25 \mathrm{sec}$ in this research). [11]. The $\mathrm{HR}_{\mathrm{ECG}}$ can thus be derived thereupon.

For $\mathrm{HR}_{\mathrm{PPG}}$, a neighboring peak searching method (as depicted in Figure 1) is adopted to derive the peak events of PPG signal in this research. In this method, two windows of 0.6 -sec width with a $0.04-\mathrm{sec}$ shift between them are selected empirically for peak searching. The PPG signal value at beginning of window 2 (the dashed one in Figure 1) is taken for a reference point (A). Moving along the PPG pattern from the reference point $\mathrm{A}$, any value greater than the reference is selected as the candidate of peak. The peak is the greatest one in all candidates found in window 2 . The two windows are then moved to next segment for another peak searching. The interval between the detected peaks is defined as the peak-to-peak interval (PPI). $\mathrm{HR}_{\mathrm{PPG}}$ is then acquired with the same procedure as that described above for the derivation of $\mathrm{HR}_{\mathrm{ECG}}$. All of the positions for derived $\mathrm{R}$ (from ECG) and P (from PPG) have been verified iteratively in this study.

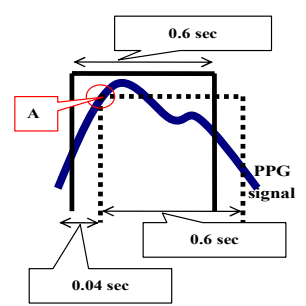

Figure 1 Depiction for peak searching of PPG signal.

\subsection{Quantities for comparison}

Having derived RRI and PPI sequences, the correlation analysis is then executed to see whether these two sequences acquired simultaneously in the same subject are highly correlated or not [12]. Let $\mathrm{x}[\mathrm{n}]$ denote the PPI sequence and $\mathrm{y}[\mathrm{n}]$ the RRI at time index n. $\bar{x}$ and $\bar{y}$ represent the mean value of $\mathrm{x}[\cdot]$ and $\mathrm{y}[\cdot]$, respectively. Then the correlation coefficient (CC) is derived according to equation (1)

$$
C C=\frac{\sum_{n=0}^{N-1}(x(n)-\bar{x})(y(n-k)-\bar{y})}{\sqrt{\sum_{n=0}^{N-1}(x(n)-\bar{x})^{2} \sum_{n=0}^{N-1}(y(n-k)-\bar{y})^{2}}} .
$$

The ratio of $\mathrm{LF} / \mathrm{HF}$ spectral power of heart rate reflects cardiac autonomic balance and has been used extensively in literatures [1]. In order to evaluate the spectral power at LF $(0.04-0.15 \mathrm{~Hz})$ and $\mathrm{HF} \quad(0.15-0.4 \mathrm{~Hz})$ band, autoregressive (AR) spectral estimation is used in our study. The AR power spectrum density (PSD) at frequency $f$ could be estimated according to the following expression

$$
p(f)=\frac{\sigma^{2} \cdot \Delta t}{\left|1+\sum_{k=1}^{p} a_{k} \cdot e^{-j 2 \pi \cdot f \cdot k \cdot \Delta t}\right|^{2}} .
$$

In which the AR coefficients $\left(a_{1}\right.$ to $\left.a_{p}\right)$ and the output power of the prediction error filter $\left(\sigma^{2}\right)$ are obtained by Burg's algorithm [13] with a fixed order 20 (i.e. $p=20$ in the above equation), $\Delta t$ is the resampling interval of the RRI and PPI sequences (which is $0.25 \mathrm{sec}$ in this research). The LF and HF power are taken as the dominant peak component within the corresponding LF $\mathrm{HF}$ band (as depicted in Figure 2).

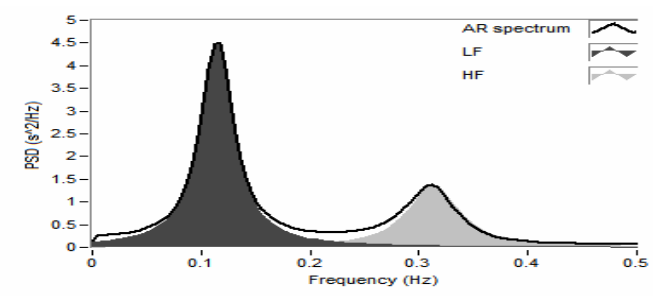

Figure 2 Power spectrum density of heart rate with the dominant LF and HF component being illustrated

Assuming that the probability distribution of $\mathrm{LF} / \mathrm{HF}$ ratio is Gaussian for the same population, the probability 
density functions $(p d f)$ for those derived from $\mathrm{HR}_{\mathrm{ECG}}$ and $\mathrm{HR}_{\mathrm{PPG}}$ can then be put together for comparison (as shown in Figure 3). Giving a threshold value on the axis of $\mathrm{LF} / \mathrm{HF}$ ratio and taking $\mathrm{HR}_{\mathrm{ECG}}$ as a standard measure for heart rate, we can derive true positive (Tp), false positive $(\mathrm{Fp})$, false negative $(\mathrm{Fn})$ and true negative $(\mathrm{Tn})$ test results as depicted in Table 1 , in which $\mathrm{A}, \mathrm{B}, \mathrm{C}$ and $\mathrm{D}$ denote the areas corresponding to the Tp, Fp, Fn and Tn results under certain threshold value. For highly approximated probability distributions as those in Figure 3 , the likelihood ratio (LR) can be used to indicate the resemblance between them. The LR can be derived according to the following equation

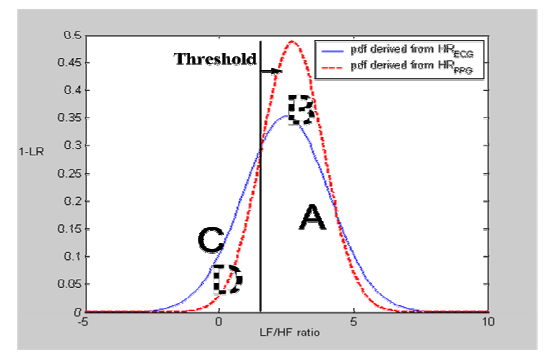

Figure 3 The depiction for the calculation of likelihood ratio (LR).

Table 1 Test results under certain decision threshold.

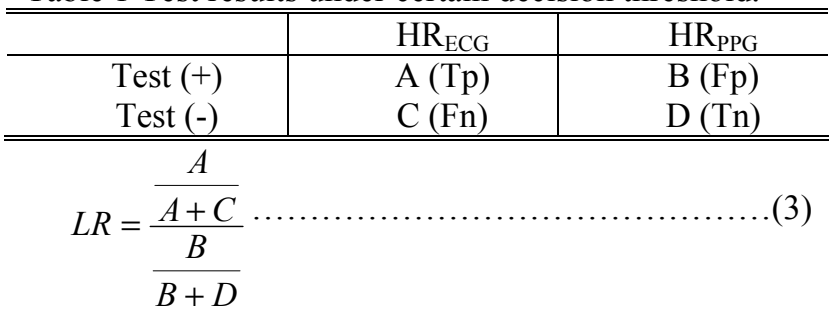

If the two distributions are the same, then $\mathrm{A}=\mathrm{B}$ and $\mathrm{C}=\mathrm{D}$ for any threshold value and a unity LR can thus be derived. Therefore, the value of (1 - LR) is used to evaluate the deviation between the distributions of LF/HF ratio derived from $\mathrm{HR}_{\mathrm{ECG}}$ and $\mathrm{HR}_{\mathrm{PPG}}$ in this research. Describe your methods here.

\section{Results}

PPI and RRI sequences for all subjects are derived from the measured raw ECG and PPG signals according to the methods described in previous section. In essence, the synchronous RRI and PPI sequence derived from the same subject should resemble much each other. It can be appreciated there exists little difference between the RRI and PPI sequence demonstrated in Figure 4. Such difference can be distinguished more easily by its scatter diagram as shown in Figure 5, which should be a straight line with a unity slope for two identical sequences.

The correlation coefficients (CC) for all of the derived
RRI and PPI sequences are summarized in Table 2. It is appreciated that some CCs are not as high as imagined (subjects No.04, No.05 and No.10). There have also been reports on the prevalence of interference on PPG signals due to the contact pressure of PPG sensor and due to the relative motion during measurement [8]. In such case, the PPG signals may be seriously degraded. For such possibilities, all of the PPG signals have been checked iteratively to assure the signal quality. As the peaks of PPG signal are much smoother than those in QRS complexes of ECG signal, this property makes the real peak not so apparent to locate especially for those PPG signals with smaller amplitude or those contaminated with high-frequency interference. In addition, there may be beat-to-beat variations of the isovolumic contraction period of the left ventricle. These may be the reasons of low CCs for these cases.

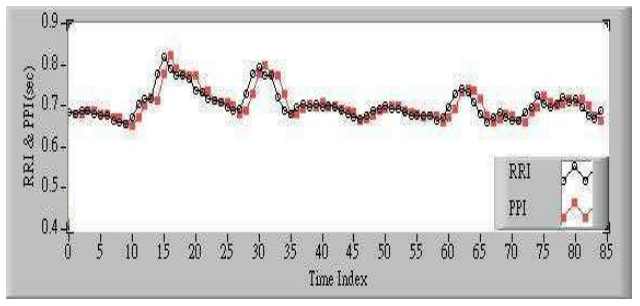

Figure 4 One example of RRI and PPI sequence (derived from subject No.06).

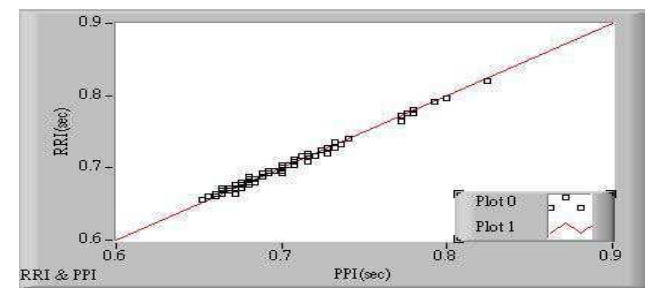

Figure 5 One scatter diagram for RRI versus PPI (for subject No.06).

Table 2 Correlation coefficients of RRI and PPI for each subject in this research.

\begin{tabular}{|c|c|}
\hline Subject & $\mathrm{CC}$ \\
\hline No.01 & 0.91 \\
\hline No.02 & 0.92 \\
\hline N0.03 & 0.98 \\
\hline No.04 & 0.71 \\
\hline No.05 & 0.58 \\
\hline No.06 & 0.99 \\
\hline No.07 & 0.95 \\
\hline No.08 & 0.96 \\
\hline No.09 & 0.97 \\
\hline No.10 & 0.65 \\
\hline Mean \pm SD & $0.86 \pm 0.15$ \\
\hline
\end{tabular}


Both the probability distributions of $\mathrm{LF} / \mathrm{HF}$ ratio derived from $\mathrm{HR}_{\mathrm{ECG}}$ and $\mathrm{HR}_{\mathrm{PPG}}$ in the same population are assumed to be Gaussian. The distributions in this frequency-domain feature are somewhat different, yet the deviation is little. As described in the section 2.3. (Quantities for comparison), the likelihood ratio (LR) can be derived for certain threshold value according to equation (3). Sliding the threshold value from the leftmost to the rightmost value of $\mathrm{LF} / \mathrm{HF}$ ratio, various LR values can thus be derived. As ( 1 - LR) may be used to evaluate the deviation between two distributions, the deviation of LR for various threshold values can be obtained as shown in Figure 6. The maximum deviation of LR is $19.04 \%$ in our research. There still needs further investigation to figure out what extent of deviation may lead to a statistical significance between two HR measurements. However, there indeed exists difference in both time-domain and frequency-domain characteristics for different approaches of heart rate measurement.

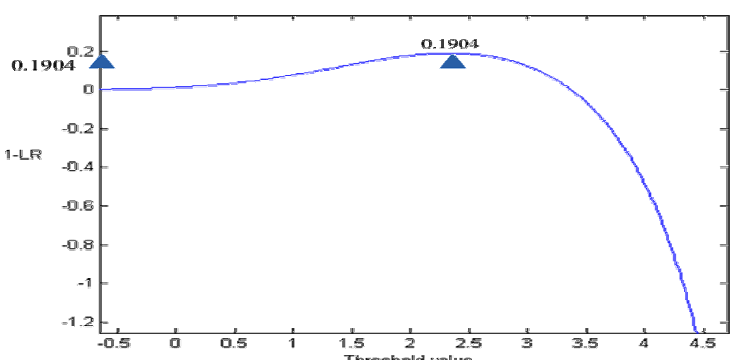

Figure 6 The deviation of likelihood ratio (LR) for various threshold values.

\section{Discussion and conclusions}

This study investigated whether any difference exists between $\mathrm{HR}_{\mathrm{ECG}}$ and $\mathrm{HR}_{\mathrm{PPG}}$ or not. Extensive experiments have been done to demonstrate the timing difference. The results illustrate there indeed exist difference between these two approaches for heart rate in both time domain (the correlation between RRI and PPI sequence) and frequency domain (the LR deviation for $\mathrm{LF} / \mathrm{HF}$ ratio derived from $\mathrm{HR}_{\mathrm{ECG}}$ and $\mathrm{HR}_{\mathrm{PPG}}$ ).

The measurement of PPG signal is convenient. Also, PPG signal contains lots of physiological significances, therefore the results of this research may help extending the applications of PPG in clinical diagnosis.

\section{Acknowledgements}

The authors would like to thank the National Science Council, Taiwan, for supporting this research under Contract No. NSC95-2221-E-035-003.

\section{References}

[1] Task Force of the European Society of Cardiology and the North American Society of Pacing and Electrophysiology. Heart rate variability: standards of measurement, physiological interpretation, and clinical use. Circulation. 1996; 93: 1043-1065.

[2] Khanokh B, Slovik Y, Landau D, Nitzan M. Sympathetically induced spontaneous fluctuations of the photoplethysmographic signal. Med \& Bio Eng \& Computing 2004; 42:80-85.

[3] Burton AC. The range and variability of the blood flow in the human fingers and the vasomotor regulation by body temperature. The American Journal of Physiology 1939; 127:473-453.

[4] Nitzan M, Babchenko A, Shemesh D, Alberton J. Influence of thoracic sympathectomy on cardiac induced oscillations in tissue blood volume. Med \& Bio Eng \& Computing 2001;39:579-583.

[5] Johansson A, Öberg PĂ. Estimation of respiratory volumes from the photoplethysmographic signal. Part 1 : experimental result. Med \& Bio Eng \& Computing 1999;37:42-47.

[6] Nakajima K, Tamura T, Miike H, Öberg PǍ. Photoplethysmographic measurement of heart and respiratory rates using digital filters. Eng. in Med. and Bio. Society Proceedings of the 15 th Annual International Conference of the IEEE, 1993;1006 - 1007.

[7] Gu YY, Zhang YT. Reducing the influence of contacting force applied on photoplethysmographic sensor on heart rate variability estimation. Proceedings of the 25th Annual International Conference of the IEEE EMBS, 2003;26182620.

[8] Teng XF, Zhang YT. The effect of contacting force on photoplethysmographic signals. Physiol. Meas.;1323-1335.

[9] Webster JG. Medical Instrumentation Application and Design. In: 3rd edition, John Wiley \& Sons Inc, 1998;368.

[10] So HH, Chan KL. Development of QRS Detection Method for Real-Time Ambulatory Cardiac Monitor. 19th International Conference on IEEE/EMBS 1997;2:289-292.

[11] Berger RD, Akselrod S, Gordon D, Cohen RJ. An efficient algorithm for spectral analysis of heart rate variability. IEEE Trans. on Biomed. Eng. 1986;33:900-904.

[12] Rangayyan RM. Biomedical signal analysis. John Wiley \& Sons Inc, 2002;95.

[13] Kay SM. Modern Spectral Estimation: Theory and Application. Prentice Hall, 1988;230-231.

[14] Van Trees HL. Detection, Estimation, and Modulation Theory Part I. John Wiley \& Sons, Inc(electronic resource) 2001;26-27.

\section{Address for correspondence}

Name: Yue-Der Lin

Full postal address: Department of Automatic Control Engineering, Feng Chia University, 100 Wenhwa Rd., Taichung City, 40724 Taiwan

E-mail address: ydlin@fcu.edu.tw 